\title{
Male foraging efficiency, but not male problem-solving performance, influences female mating preferences in zebra finches
}

\author{
Véronique Chantal ${ }^{1}{ }$, Julie Gibelli $^{1}{ }^{\text {, Frédérique Dubois }}{ }^{\text {Corresp. }}{ }^{1}$ \\ ${ }^{1}$ Département de sciences biologiques, Université de Montréal, Montréal, Canada \\ Corresponding Author: Frédérique Dubois \\ Email address: frederique.dubois@umontreal.ca
}

Experimental evidence suggests that females would prefer males with better cognitive abilities as mates. However, little is known about the traits reflecting enhanced cognitive skills on which females might base their mate-choice decisions. In particular, it has been suggested that male foraging performance could be used as an indicator of cognitive capacity, but convincing evidence for this hypothesis is still lacking. In the present study, we investigated whether female zebra finches (Taeniopygia guttata) modify their mating preferences after having observed the performance of males on a problem-solving task. Specifically, we measured the females' preferences between two males once before and once after an observation period, during which their initially preferred male was incapable of solving the task contrary to their initially less-preferred male. We also conducted a control treatment to test whether the shift in female preferences was attributable to differences between the two stimulus males in their foraging efficiency. Finally, we assessed each bird's performance in a color associative task to check whether females can discriminate among males based on their learning speed. We found that females significantly increased their preference toward the most efficient male in both treatments. Yet, there was no difference between the two treatments and we found no evidence that females assess male cognitive ability indirectly via morphological traits. Thus, our results suggest that females would not use the males' problem-solving performance as an indicator of general cognitive ability to gain indirect fitness benefits (i.e., good genes) but rather to assess their foraging efficiency and gain direct benefits. 
1 Male foraging efficiency, but not male problem-solving performance, influences female

2

3

4

5

6

7

8 Running title: Feeding success affects mate preferences

9

10 Corresponding author:

11 Frédérique Dubois, Département de Sciences Biologiques, Université de Montréal, CP 6128

12 Succ. Centre-ville, Montréal Qc, H3C3J7 Canada

13 Tel: (1) 514-343-6927

14 Email: frederique.dubois@umontreal.ca 


\section{ABSTRACT}

17 Experimental evidence suggests that females would prefer males with better cognitive abilities as mates. However, little is known about the traits reflecting enhanced cognitive skills on which

19 females might base their mate-choice decisions. In particular, it has been suggested that male foraging performance could be used as an indicator of cognitive capacity, but convincing evidence for this hypothesis is still lacking. In the present study, we investigated whether female zebra finches (Taeniopygia guttata) modify their mating preferences after having observed the performance of males on a problem-solving task. Specifically, we measured the females' preferences between two males once before and once after an observation period, during which their initially preferred male was incapable of solving the task contrary to their initially lesspreferred male. We also conducted a control treatment to test whether the shift in female preferences was attributable to differences between the two stimulus males in their foraging efficiency. Finally, we assessed each bird's performance in a color associative task to check whether females can discriminate among males based on their learning speed. We found that females significantly increased their preference toward the most efficient male in both treatments. Yet, there was no difference between the two treatments and we found no evidence that females assess male cognitive ability indirectly via morphological traits. Thus, our results suggest that females would not use the males' problem-solving performance as an indicator of general cognitive ability to gain indirect fitness benefits (i.e., good genes) but rather to assess their foraging efficiency and gain direct benefits. 


\section{INTRODUCTION}

38 As the brain structures needed to acquire, process, store and use information from the environment are costly to develop and maintain, cognitive abilities in both humans and animals are often considered as an honest indicator of genetic quality that should be used as a matechoice criterion (Jacobs 1996; Miller 2000; Boogert et al. 2011a). More precisely, improved cognitive abilities can help animals to respond quickly and adequately to environmental changes (Kotrschal and Taborsky 2010). Females, therefore, might benefit from choosing a mate with higher cognitive ability because it would be better to cope with changing conditions, hence providing them and their offspring with better resources. Females could also gain indirect benefits when the cognitive traits are heritable (Croston et al. 2015). Supporting the idea that males with better cognitive skills are preferred as mates, two studies have demonstrated that males with better spatial learning abilities are more attractive to females in both meadow voles (Microtus pennsylvanicus) (Spritzer et al. 2005) and guppies (Poecilia reticulata) (Shohet and Watt 2009). Also there is good evidence that birdsong, which is an indicator of brain development (Farrell et al. 2015), plays an important role in mate attraction (Searcy and Andersson 1986; Nowicki et al. 2002). Yet, relatively few studies except those concerning song learning in birds, have looked at how individuals assess the cognitive capacity of the opposite sex. Consequently, little is known about the traits reflecting enhanced cognitive skills on which females might base their mate-choice decisions in other taxa or even in bird species in which song complexity is not a meaningful indicator of cognitive capacity (Boogert et al. 2011b;

57 Templeton et al. 2014). females would use as an indicator of cognitive capacity (Boogert et al. 2011a). In particular, 
60 females could discriminate among males based on their ability to solve novel problems. Indeed,

61

62

63

64 experimental evidence has shown that individuals of the same population may differ widely in their problem-solving success and that this trait correlates positively with performance on various learning tasks (Bouchard et al. 2007; Boogert et al. 2008; Cole et al. 2011; Overington et al. 2011; Aplin et al. 2013; Griffin et al. 2013; Templeton et al. 2014; Shaw et al. 2015). These findings suggest that males with better problem-solving ability would have higher general cognitive ability (Shaw et al. 2015). In addition, recent research has established a link between problem-solving ability and mating success, hence providing evidence that females would also obtain direct fitness benefits from choosing mates with better cognitive skills. Specifically, Keagy et al. (2009, 2011) have reported that male satin bowerbirds (Ptilonorhynchus violaceus) with better problem-solver ability in the field obtain more copulations, while two recent studies on great tits (Parus major) have demonstrated that more cognitively skilled mates that are faster problem solvers produce more offspring (Cole et al. 2012; Cauchard et al. 2013).

To date, however, evidence for the hypothesis that females use male foraging performance as an indicator of cognitive ability is indirect. Indeed, several studies in birds (Hill 1990) and fish (Pike et al. 2007) have reported that females prefer brighter or more colored males, probably because they are more efficient in acquiring food and hence ingest more carotenoids responsible for brightly colored sexual ornaments. Yet, it is unclear whether carotenoid coloration reflects male foraging success and whether females use direct observation of male cognitive performance rather than traits that are correlated with cognition when choosing a mate. Only Snowberg and Benkman (2009) have demonstrated that female crossbills (Loxi curvirostra) rely on male foraging performance to choose a mate. More precisely, they found that females that had observed two males that differed in their feeding rate preferred the most efficient one. However, 
83 there is no evidence that male crossbills that are more efficient at extracting seeds from conifer

84 cones have better cognitive skills. Therefore, no study has yet directly tested whether females

85 discriminate among males through direct observation of their performance on a foraging task that

86 indicates cognitively ability.

87 In the present study, we addressed this question by investigating whether female zebra

88 finches (Taeniopygia guttata) modify their mating preferences after having observed the

89 foraging performance of males on a problem-solving task. Although male song has been found to

90 be important for female choice in this species (Riebel 2009), recent findings indicate that song

91 complexity would not be a good indicator of general cognitive ability (Templeton et al. 2014), as

92 previously thought (Boogert et al. 2008). Females, therefore, might benefit from using other cues

93 that best reflect a male's overall cognitive ability, such as its ability to solve novel problems.

94 Thus, to assess the influence of this cue on female mate-choice decisions, we trained males to

95 solve a task, and then we measured the mating preferences of each female twice: before and after

96 she had observed the performance of two stimulus males on the task (main treatment). We

97 experimentally manipulated the performance of the two males during the observation period, so

98 that each female could observe her initially preferred male that was incapable of solving the task

99 (i.e., the non solver) and her initially less-preferred male (i.e., the solver) that, on the contrary,

100 was highly efficient at solving the task. Furthermore, because only the solver could access food,

101 we conducted a control treatment to test whether the change in females' preferences observed in

102 the main treatment could be explained by differences among males in their foraging efficiency

103 rather than in their ability to solve the task. Finally, we measured each bird's learning

104 performance in a color associative task in order to check 1) whether females, prior to the

105 observation period, could discriminate between the two males based on their learning 
106 performance and hence preferred the male that learned faster, and 2) whether the ability of

107 females to assess male cognitive ability was related to their own learning performance.

108

109 METHODS

110 (a) Subjects and housing

111 We used 40 (30 females and 10 males) commercially purchased unrelated adult zebra finches

112 obtained from a local breeder (Exotic Wings \& Pet Things, St Clements, Ontario, Canada).

113 Twenty-two birds (18 females and 4 males) and 20 birds (12 females and 6 males) were used in

114 the main and control treatments, respectively. Outside the testing periods, the birds were kept in 115 groups of two or three in same-sex cages $(10 \times 40 \times 30 \mathrm{~cm})$ with a 14: 10 hour light: dark

116 photoperiod at approximately $23 \pm 1{ }^{\circ} \mathrm{C}$. They had ad libitum access to seeds, water and cuttlefish

117 bone. In addition, their diet was supplemented once a week with egg yolk mixture and

118 vegetables. The experiments described in this study were approved by the Animal Care

119 Committee of the University of Montreal (animal care permit \#14-073) and conformed to all 120 guidelines of the Canadian Council on Animal Care.

(b) Main treatment

\section{Problem-solving task}

124 Before we measured the preferences of each female between one solver and one non solver, we 125 trained the males to solve a task, which consisted of a transparent plastic tube filled with millet 126 seeds and closed with a lid that the bird had to flip to get access to the food (Fig. 1). Training 127 sessions occurred between 7 h00 and 13 h00 after overnight food deprivation and lasted for 20 128 consecutive days with two sessions per day separated by 3 hours. Males were trained by pairs in 
129 their housing cage that was divided by an opaque partition in two sections. Thus, the birds could

130 not scrounge food or observe each other's behavior. The day before the training began, we

131 placed two apparati outside of the cage, to allow the birds to become familiar with them. Then

132 the training procedure consisted of the following three steps: 1) we provided the birds with an

133 open tube (i.e., with no lid); 2) once the birds had eaten for $10 \mathrm{sec}$ in step 1, a lid was just

134 deposited on the tube, so that the birds could easily get access to the food by pushing down the

135 lid. An individual who succeeded in pushing down the lid had access to the food during $10 \mathrm{sec}$

136 before the lid was replaced; 3) once the birds had succeeded 5 times in step 2, the lid was pressed

137 halfway so that the birds had now to flip the lid to get access to the food. The training was over

138 when the birds could open the tube at least 10 times during a 60 min period.

Mate-choice apparatus and experimental procedure

141 We measured female mating preferences with a classical binary choice apparatus (Fig. 2) that

142 comprised three compartments: A) the observation compartment where the focal female could

143 see both males simultaneously, B) the choice compartment where she could see only one

144 stimulus male at a time and C) the male compartment divided into two identical chambers, each

145 housing a single male. Before the beginning of the experiment, males and females were placed

146 individually in the apparatus during one hour for 10 days to become familiar with their

147 environment. Then we measured each female's preference twice (i.e., initial and final

148 preferences): before and after an observation period, during which she could observe one of the

149 two stimulus males solving the task while the other did not.

150 The initial preference of each focal female was measured following this procedure: after the two

151 stimulus males had been placed in the male compartments, we introduced the test female in the 
152 observation compartment and after a $15 \mathrm{~min}$ period, we gently lifted the transparent partition

153 between the observation and choice compartments. We then measured the time she spent on the

154 perches in the neutral zone and in front of each male during two consecutive periods of $30 \mathrm{~min}$

155 each, switching the position of the males after $30 \mathrm{~min}$. To control for differences in the stimulus

156 males' songs, we masked their songs during the duration of the mate choice tests by playing a

157 recorded chorus of calls and songs from male and female zebra finches. Furthermore, to ensure

158 that the females were able to distinguish between the two males, we formed the pairs so that the

159 two stimulus males differed in terms of size, plumage and beak color.

160 The 5 days following the initial preference test, each female was placed in the observation

161 compartment for two periods per day during which she could observe the two stimulus males

162 while they were interacting with the problem-solving task. Before each observation period, the

163 two stimulus males were food deprived for 3 hours. Then, in order to manipulate their success,

164 one of them (i.e., the solver) was provided with a tube the lid of which was pressed only halfway

165 and hence that could be easily opened, while the other male (i.e., the nonsolver) was provided

166 with a tube the lid of which was fully pressed and hence that was impossible to open. For each

167 female, the easy task was provided to her initially less-preferred male while the difficult task was

168 provided to her initially preferred male. Each observation period ended after 60 min or once the

169 most efficient male had solved the task 10 consecutive times, whichever occurred first. In

170 addition, to prevent female-male interactions and other distractions during the observation

171 periods, we placed three natural-spectrum, $60 \mathrm{~W}$ light bulbs directly in front of each male's

172 compartment, making it harder for the males in their brightly lit environment to detect the female

173 in her shaded compartment in front of them. 
After 24h, we measured the final preference of the focal female using the same procedure

175

176

177

178

179

180

181

182

183

184

185

186

187

188

189

190

191

192

193

194

195

196

as for the initial tests. All females, except one female that was injured after the initial test, were tested for their final preferences. In addition, although females observed the performance of the males on the problem-solving task only during the observation sessions, males were provided with the task every day during habituation and testing periods, so that they do not forget how to flip the lid.

\section{(c) Control treatment}

We used exactly the same procedure as described above, except for the observation period, during which the test female could observe twice a day the two stimulus males while they were searching for seeds within a dish $(13 \times 7 \times 3.5 \mathrm{~cm})$. The food dishes that were provided to the two stimulus males both contained a double layer of dried peas that acted as obstacles, thereby forcing the birds to move them around to detect and gain access to the millet seeds when they were present. In addition, in order to manipulate the feeding rate of the two stimulus males, the initially preferred male was provided a dish that contained no seeds while the other male was provided a dish with 30 millet seeds. Each observation period ended after 10 min or 2 min after the most efficient male had stopped searching for food, whichever occurred first.

\section{(d) Associative learning task}

We measured the performance of all individuals (i.e., both males and females) in a color associative task as the number of trials needed to find 6 consecutive times the rewarded feeder. Specifically, the birds were tested individually in an experimental apparatus that comprised an observation chamber $(20 \times 50 \times 30 \mathrm{~cm})$ and a choice chamber $(40 \times 50 \times 30 \mathrm{~cm})$ that were separated 
197 from each other by a transparent removable partition. The choice chamber was divided into four

198 symmetrical corridors, and at the end of each corridor we placed 4 white feeders that were

199 positioned in front of 4 colored dots (i.e., yellow, cyan, pink and black) whose position changed

200 randomly from one trial to the next. The rewarded feeder (i.e., the feeder placed in front of the

201 yellow dot) always contained 4 millet seeds, while the other feeders were empty.

202

203 Prior to testing, we trained the birds to eat from the feeder that was deposited within their home

204 cages. Then, the birds were placed in the experimental apparatus to become familiarized with the

205 environment. They spent at least 3 hours per day for 2 weeks in the apparatus until they could

206 explore the 4 corridors and eat without fear from the feeders, whatever their position.

207

208

Before each testing day, the birds were food deprived for 3 hours. They experienced a maximum

of 25 trials per day during 4 consecutive days or until they had reached the learning criterion, whichever occurred first.

At the beginning of each trial, the bird was confined in the observation chamber for $2 \mathrm{~min}$. Then,

212 the observer gently lifted the removable partition, thereby allowing the bird to enter in the choice chamber and choose one of the 4 feeders. Once the bird had chosen a corridor, we noted whether

214 it had succeeded or failed. If the bird had succeeded, it could eat the 4 seeds before returning to 215 the observation chamber. On the contrary, if the bird had failed, the observer either gently 216 activated the removable partition to encourage the bird to return into the observation chamber if

217 it had obtained food during the previous trial or let it explore the other corridors and find the

218 rewarded feeder otherwise. Such a procedure was adopted to insure that all the birds ate 219 approximately the same amount of food during each session and that differences among 
220 individuals in their learning speed, therefore, were not due to differences in their level of satiety.

221 All but 3 injured birds ( 2 males and 1 female) were used for this experiment.

222

223 (e) Statistical analyses

224 To determine whether the females were capable of discriminating between the two stimulus

225 males based on their learning capacity, we tested whether the percentage of time spent in front of

226 the male who resolved the color association task faster was significantly larger than 50\% using a

227 one-sample t-test. Because we used 5 different pairs of males for the preference tests, we also

228 conducted a one-way ANOVA to assess whether female preferences differed among the pairs of

229 stimulus males. Next, we compared the average learning performance of the females that

230 expressed a marked preference (i.e., spent 55\% or more of their choosing time in front of one

231 male) for either the fast or the slow learning male using a t-test, and we used a Pearson

232 correlation coefficient to determine whether the relative time spent by females in front of their

233 initially preferred male was correlated with the difference between the two stimulus males in

234 their learning speed.

235

236 For both treatments, we assessed whether the change in the females' preferences (i.e., the relative

237 time spent in front of the most efficient male in the final preference test minus the relative time

238 spent in front of the same male in the initial preference test) significantly differed from zero

239 using a paired t-test, and then we performed a t-test to determine if the change in preferences

240 differed between the two treatments. We also verified that the relative time spent in the choosing

241 zone was not significantly different between the initial and final test preferences using a paired t-

242 test, and for both variables (i.e., change in the females' preferences and change in their relative 
243 time spent in the choice zone) we conducted a one-way ANOVA to test for an effect of pair

244 identity. Finally, we used Pearson's correlation coefficient to test for an association between the

245 change in females' preferences and their learning score. Data were excluded from the analyses

246 when females spent less than $30 \%$ of their time in the choosing zone. Statistical analyses were

247 done with SPSS 23.0 for Mac.

\section{RESULTS}

250 During the initial preference test, females on average $(\mathrm{X} \pm \mathrm{SE})$ spent $47.83 \pm 4.60 \%$ of their

251 choosing time in front of the faster learner of the two stimulus males in the color association

252 task, which is not significantly different from $50 \%\left(\mathrm{t}_{23}=-0.471, P=0.642\right)$. Furthermore, there was

253 no significant effect of the identity of the stimulus males on the expression of female preferences

$254\left(\mathrm{~F}_{3,20}=0.066, P=0.977\right)$. Female choice, therefore, was random with respect to male learning

255 performance in the color association task. The relative time spent by females in front of their

256 less-preferred male was not correlated either with the difference in learning speed between the

257 two stimulus males $(\mathrm{r}=-0.075, \mathrm{~N}=30, P=0.694)$. This finding indicates that females that had to

258 choose between two males that differed largely in their learning performance were not more

259 likely to prefer the faster learner of the two stimulus males than those that had to choose between

260 two potential mates with more similar learning speeds. Finally, the mean number of trials needed

261 to solve the color associative learning task was not significantly different between females that

262 preferred the faster learner and those that preferred the slower learner of the two males $\left(\mathrm{t}_{10}=-\right.$

$2630.622, P=0.548)$. 
265 The time spent by females in the choice zone was not significantly different between the initial

266 and final preference tests $\left(\mathrm{t}_{27}=-0.335, P=0.740\right)$. On the contrary, we found that females

267 significantly increased their preference toward the initial less-preferred male after having

268 observed the performance of the two stimulus males in both treatments (main treatment:

$269 \mathrm{t}_{15}=2.608, P=0.020$; control treatment: $\mathrm{t}_{11}=2.472, P=0.031$; Fig. 3). Yet, there was no significant

270 effect of the treatment on the shift in female preferences $\left(\mathrm{t}_{26}=1.164, P=0.255\right)$ and neither

271 variable was affected by the identity of the stimulus males (change in the relative time spent in

272 the choice zone: $\mathrm{F}_{4,24}=0.072, P=0.990$; change in the relative choosing time spent in front of the

273 initially less-preferred male: $\mathrm{F}_{4,23}=0.832, P=0.579$ ). Finally, we found no correlation between the

274 females' learning speed in the color association task and the magnitude of the change in their

275 mating preferences in the main treatment $(\mathrm{r}=0.178, \mathrm{~N}=16, P=0.509)$ or in the control treatment

$276(\mathrm{r}=0.269, \mathrm{~N}=11, P=0.424)$.

277

278

279

280

281

282

283

284

285

286

287

\section{DISCUSSION}

We found that zebra finch females significantly increased their mating preference toward the most efficient (initially less preferred) male, after having observed the performance of the two stimulus males in both treatments. Because in both treatments, the two stimulus males differed in their feeding rate, our results suggest that females use male foraging efficiency as a mate-choice criterion. This result is in agreement with the study of Snowberg and Benkman (2009) who reported that red crosbill females also preferred the male that was the more efficient forager. In zebra finches, variation among individuals in their feeding rate causes variation in their reproductive success (Lemon and Barth 1992; Lemon 1993). More precisely, because individuals with high rates of energy gain have more time and energy available for reproduction compared 
288 with less efficient foragers, they are able to produce more offspring that also survive better.

289 Female zebra finches, therefore, can gain direct fitness benefits from choosing mates based on 290 their foraging efficiency.

291

292 Yet, contrary to our expectations, we found no support for the hypothesis that zebra finch

293 females discriminate among potential mates based on their problem-solving ability. Indeed, we

294 detected no significant difference in the change of female preferences between the two

295 treatments, which means that the capacity of the males to solve the task in the main treatment

296 was unimportant for females compared to the males' feeding rate. Thus, our results indicate that

297 female zebra finches do not use male problem-solving performance as an indicator of cognitive

298 capacity. One reason that could explain this finding is that mate assessment based on male

299 foraging performance likely requires considerable time, which would prevent most females from

300 using this trait as a mate-choice criterion. Indeed, as zebra finches are opportunistic breeders,

301 starting to breed immediately after rain (Zann 1996), females have to make quick mating

302 decisions. Under natural conditions, however, the probability of observing a cognitively

303 demanding foraging behavior (e.g., an innovation) is expected to be very low. In order to reduce

304 the cost of mate assessment, females would then benefit from using morphological traits that are

305 correlated with cognitive abilities, instead of assessing directly the males' cognitive

306 performance. However, we found no evidence for this explanation.

307

308 Indeed, prior to the observation of the males' performance, females did not prefer the faster

309 learner of the two stimulus males and we found no evidence, either, that they chose assortatively

310 based on learning capacity. These findings indicate that female zebra finches do not assess male 
311 cognitive ability indirectly via morphological traits or courtship displays, irrespective of their

312 own cognitive abilities. Although it is possible that we failed to detect a preference of females

313 for the faster learner of the two stimulus males because there was not enough variation among

314 them in their learning performance, this explanation is unlikely. Indeed, we found no correlation

315 between the strength of female preferences and the difference in learning speeds between the two

316 stimulus males, which means that the time spent by females in front of the fast-learning male

317 was not influenced by the amount of variation between the two potential mates in their cognitive

318 ability. So, our results suggest that females would not use the males' performance on different

319 learning tasks as an indicator of general cognitive ability to gain indirect fitness benefits (i.e.,

320 good genes) but rather to assess their foraging efficiency and hence gain direct fitness benefits.

321 This conclusion is supported by the fact that several authors have reported non-significant

322 correlations among individual performance on different cognitive tasks (Boogert et al. 2011b;

323 Templeton et al. 2014; Farrell et al. 2015; Kriengwatana et al. 2015), which strongly suggests

324 that different cognitive measures would each reflect a specific ability. As a consequence, though

325 our results need to be further confirmed, we argue that cognitive traits could evolve through

326 sexual selection only if enhanced cognitive skills enable males to acquire more resources and

327 hence to produce more viable offspring.

329 In conclusion, our results showed that female zebra finches use direct observation of foraging

330 efficiency to guide their mate-choice decisions, probably because females mated with highly

331 efficient foragers are able to produce more offspring that survive better. Yet, we found no

332 evidence that females assess males' cognitively ability either directly via observation of their

333 performance on a problem-solving task or indirectly via morphological traits that are correlated 
334 with their learning ability. Thus, our results do not support the hypothesis that female zebra

335 finches would use male learning ability as an indicator of general cognitive ability, but additional

336 studies would be required to confirm our conclusions. In particular, given that male song is an

337 important mate-choice criterion used by females, future studies should explore whether song

338 advertises direct benefits, indirect benefits or both (Farrell et al. 2015).

ACKNOWLEDGEMENTS

341

342

343

344

345

346

347

We thank Guillaume Pilon for his help during data collection.

\section{REFERENCES}

Aplin LM, Sheldon BC, Morand-Ferron J (2013) Milk bottles revisited: social learning and individual variation in the blue tit, Cyanistes caeruleus. Anim Behav 85: 1225-1232

Boogert NJ, Anderson RC, Peters S, Searcy WA, Nowicki S (2011b) Song repertoire size in male house sparrows correlates with detour reaching, but not with other cognitive measures. Anim Behav 81: 1209-1216

Boogert NJ, Fawcett TW, Lefebvre L (2011a) Mate choice for cognitive traits: a review of the evidence in nonhuman vertebrates. Behav Ecol 22: 447-459

Boogert NJ, Giraldeau L-A, Lefebvre L (2008) Song complexity correlates with learning ability in zebra finch males. Anim Behav 76: 1735-1741

Bouchard J, Goodyer W, Lefebvre L (2007) Social learning and innovation are positively correlated in pigeons (Columba livia). Anim Cogn 10: 259-266

Cauchard L, Boogert NJ, Lefebvre L, Dubois F, Doligez B (2013) Problem-solving performance is correlated with reproductive success in a wild bird population. Anim Behav 85: 19-26 
357 Cole EF, Cram DL, Quinn JL (2011) Individual variation in spontaneous problem-solving

358 performance among wild great tits. Anim Behav 81: 491-498

359 Cole EF, Morand-Ferron J, Hinks AE, Quinn JL (2012) Cognitive ability influences reproductive

360 life history variation in the wild. Curr. Biol. 22: 1808-1812

361 Croston R, Branch CL, Kozlovsky DY, Dukas R, Pravosudov VV (2015) Heritability and the

362 evolution of cognitive traits. Behav. Ecol. 26:1447-1449

363 Farrell T, Kriengwatana B, MacDougall-Shackleton SA (2015) Developmental stress and

364 correlated cognitive traits in songbirds. Comp Cogn Behav Rev 10: 1-23

365 Griffin AS, Guez D, Lermite F, Patience M (2013) Tracking changing environments: innovators

366 are fast, but not flexible learners. PLoS One 8: e84907

367 Hill GE (1990) Female house finch prefer colourful males: sexual selection for condition-

368 dependent trait. Anim Behav 40: 563-572

369 Jacobs LF (1996) Sexual selection and the brain. Trends Ecol Evol 11: 82-86

370 Keagy J, Savard JF, Borgia G (2009) Male satin bowerbird problem-solving ability predicts

371 mating success. Anim Behav 78: 809-817

372 Kotrschal A, Taborsky B (2010) Environmental change enhances cognitive abilities in fish. Plos

$373 \quad$ Biol. 8: e1000351

374 Kriengwatana B, Farrell TM, Aitken SDT, Garcia L, MacDougall-Shackleton SA (2015) Early-

375 life nutritional stress affects associative learning and spatial memory but not performance on a

376 novel object test. Behaviour 152: 195-218

377 Lemon WC (1993) The energetics of lifetime reproductive success in the Zebra finch

378 Taeniopygia guttata. Physiol Zool 66: 946-963

379 Lemon WC, Barth RH (1992) The effect of feeding rate on reproductive success in the zebra 
380

381

382

383

384

385

386

387

388

389

390

391

392

393

394

395

396

397

398

399

400

401

finch, Taeniopygia guttata. Anim Behav 44: 851-857

Miller GF (2000) The mating mind: how sexual choice shaped the evolution of human nature.

$$
\text { Doubleday, New York }
$$

Nowicki S, Searcy WA, Peters S (2002) Brain development, song learning and mate choice in birds: a review and experimental test of the nutritional stress hypothesis. J Comp Physiol A

$$
\text { 188: } 1003-1014
$$

Overington SE, Cauchard L, Côté KA, Lefebvre L (2011) Innovative foraging behaviour in birds: what characterizes an innovator? Behav Process 87: 274-285

Pike TW, Blount JD, Bjerkeng B, Lindstrom J, Metcalfe NB (2007) Carotenoids, oxidative stress and female mating preference for longer lived males, Proc R Soc Lond B Biol Sci 274: 1591-

$$
1596
$$

Riebel K (2009) Song and female choice in zebra finches: a review. Adv Stud Behav 40: 197238

Searcy WA, Anderson M (1986) Sexual selection and the evolution of song. Annu Rev Ecol Syst 17: $507-533$

Shohet AJ, Watt PJ (2009) Female guppies Poecilia reticulata prefer males that can learn fast. J Fish Biol 75: 1323-1330

Snowberg LK, Benkman CW (2009) Mate choice based on a key ecological performance trait. J Evol Biol 22: 762-769

Shaw RC, Boogert NJ, Clayton NS, Burns KC (2015) Wild psychometrics: evidence for 'general' cognitive performance in wild New Zealand robins, Petroica longipes. Anim Behav 109: $101-111$ 
402 Spritzer MD, Meikle DB, Solomon NG (2005) Female choice based on male spatial ability and 403 aggressiveness among meadow voles. Anim Behav 69: 1121-1130

404 Templeton CN, Laland KN, Boogert NJ (2014) Does song complexity correlate with problem405 solving performance in flocks of zebra finches? Anim Behav 92: 63-71

406 Zann RA (1996) The Zebra Finches: a synthesis of field and laboratory studies. Oxford

407 University Press, Oxford 


\section{FIGURE LEGENDS}

410 Fig. 1. Side view of the motor learning task. The lid of the plastic tube was pressed either

411 halfway to allow the bird to easily flip the lid or fully pressed to prevent the bird to get access

412 to the food.

413 Fig. 2. Top view of the mate-choice apparatus with: the observation compartment (A), the male 414 compartment (D) and the choice compartment divided into the neutral zone (B) and the choice 415 zone (C). The grey lines represent the perches while the black lines correspond to the 416 partitions that were opaque (full lines) or clear (dashed lines).

417 Fig 3. Mean $( \pm$ SEM) percent of choosing time spent in front of the male that was the most

418 efficient forager, before and after females had observed the males' performance in the main 419 (white bars) and control (grey bars) treatments. 


\section{Figure 1}
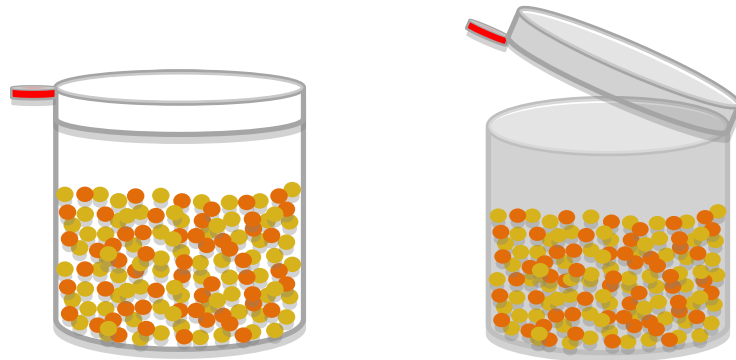

422

A

B

423 
424 Figure 2

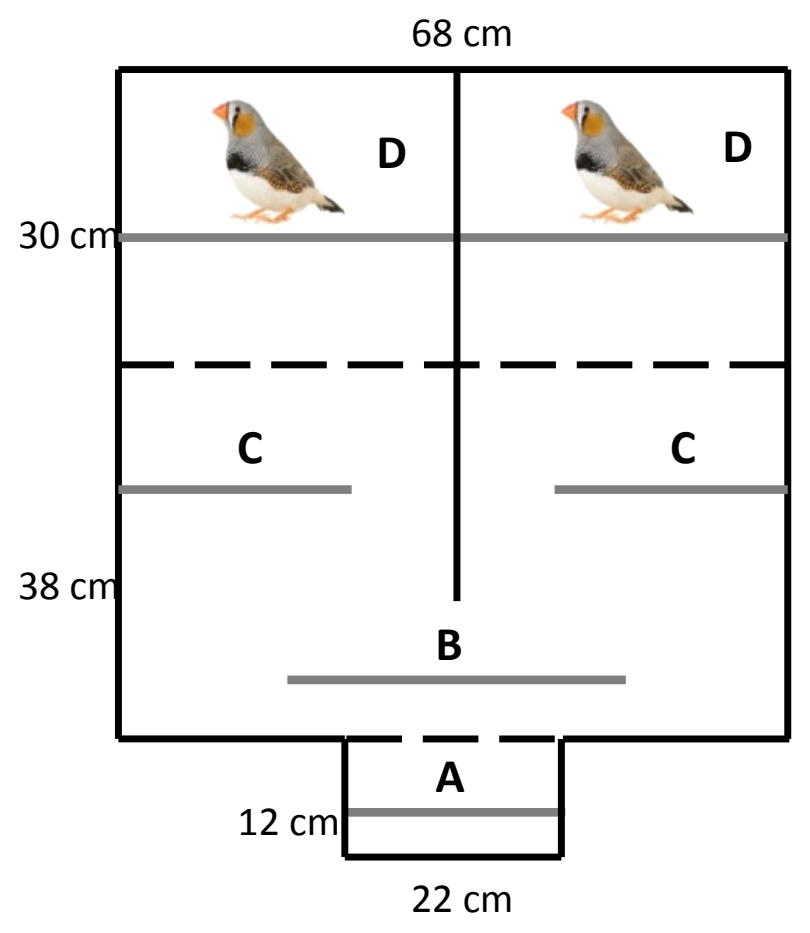

425

426

427 
428 Figure 3

429

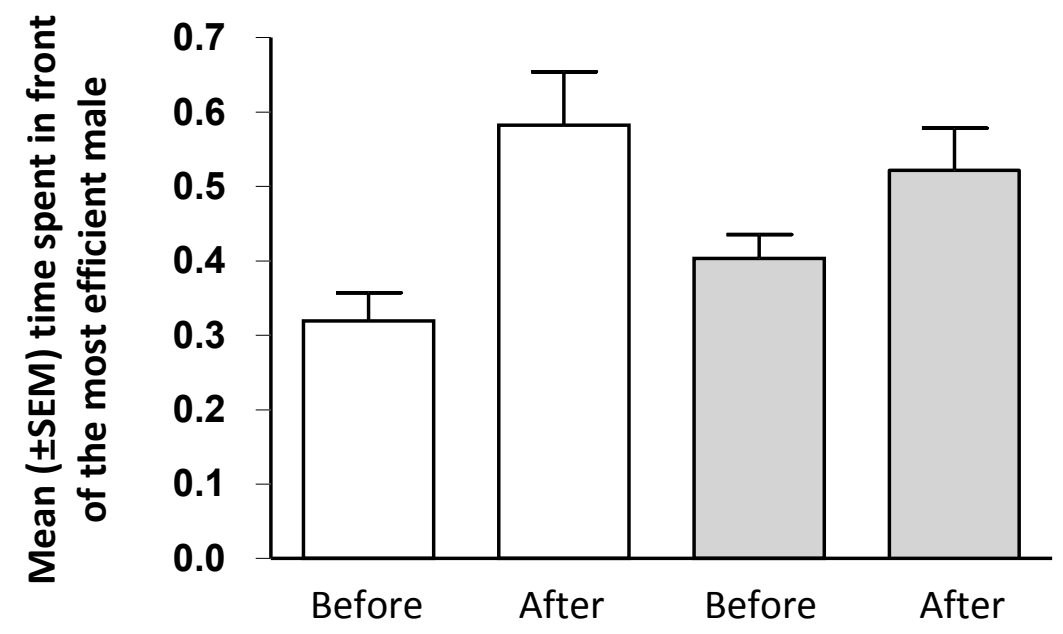

